\title{
Structure formation in atom lithography using geometric collimation
}

\author{
T. Meijer · J.P. Beardmore • C.G.C.H.M. Fabrie • \\ J.P. van Lieshout • R.P.M.J.W. Notermans • R.T. Sang • \\ E.J.D. Vredenbregt $\cdot$ K.A.H. van Leeuwen
}

Received: 16 April 2011 / Revised version: 8 July 2011 / Published online: 12 October 2011

(C) The Author(s) 2011. This article is published with open access at Springerlink.com

\begin{abstract}
Atom lithography uses standing wave light fields as arrays of lenses to focus neutral atom beams into line patterns on a substrate. Laser cooled atom beams are commonly used, but an atom beam source with a small opening placed at a large distance from a substrate creates atom beams which are locally geometrically collimated on the substrate. These beams have local offset angles with respect to the substrate. We show that this affects the height, width, shape, and position of the created structures. We find that simulated effects are partially obscured in experiments by substrate-dependent diffusion of atoms, while scattering and interference just above the substrate limit the quality of the standing wave lens. We find that in atom lithography without laser cooling the atom beam source geometry is imaged onto the substrate by the standing wave lens. We therefore propose using structured atom beam sources to image more complex patterns on subwavelength scales in a massively parallel way.
\end{abstract}

\section{Introduction}

Atom lithography is a technique to structure atomic depositions, based on the use of dipole interactions of neutral

T. Meijer · C.G.C.H.M. Fabrie · J.P. van Lieshout .

R.P.M.J.W. Notermans · E.J.D. Vredenbregt ·

K.A.H. van Leeuwen ( $\varangle)$

Department of Applied Physics,

Eindhoven University of Technology, P.O. Box 513,

5600 MB Eindhoven, The Netherlands

e-mail: K.A.H.v.Leeuwen@ tue.nl

J.P. Beardmore $\cdot$ R.T. Sang

Centre for Quantum Dynamics, Griffith University, Nathan,

Queensland 4111, Australia atoms with near-resonant light fields. As the dipolar interaction energy is intensity dependent, a standing wave light field leads to a periodic potential. This potential can be used as an array of lenses for neutral atoms, with a periodicity of $\lambda / 2$, where $\lambda$ is the wavelength of the light. Pioneering experiments were performed by Timp et al. using sodium atoms [1] and by McClelland et al. using chromium [2]. In these experiments, beams of atoms were transversely laser cooled and then focussed on a substrate into nanolines spaced by exactly $\lambda / 2$. Atom lithography experiments were also performed with other elements: $\mathrm{Al} \mathrm{[3],} \mathrm{Yb}$ [4], and Fe $[5,6]$. Experiments using light fields other than a onedimensional standing wave have been used to create structures such as rectangular grids [7], hexagonal arrays [8], quasi-periodic patterns [9], or holographically created patterns [10]. The quantum mechanical nature of the interaction of neutral atoms and resonant light allows for the creation of structures of $\lambda / 4$ [11] or $\lambda / 8$ periodicity [12]. Reviews of atom lithography are given in [13, 14].

In previous atom lithography experiments, atomic beams were transversely laser cooled to increase beam brightness and to minimise transverse velocity spread. The use of laser cooling can increase brightness by orders of magnitude [15], but the brightness increase is much lower in many setups. Atomic species that lack a closed atomic transition from the ground state, e.g. Al, Ga, or In [16], are not suitable for laser cooling in atom lithography experiments as atoms that decay to different atomic states no longer interact with the light fields. Complex laser cooling schemes can be applied, but these are experimentally challenging. The second reason for laser cooling, minimising the transverse velocity spread, was thought to be a key requirement for atom lithography [13], but was shown not to be essential by Smeets et al. [17].

For atom lithography the local transverse velocity spread of an atom beam impinging on a single-atom lens needs 


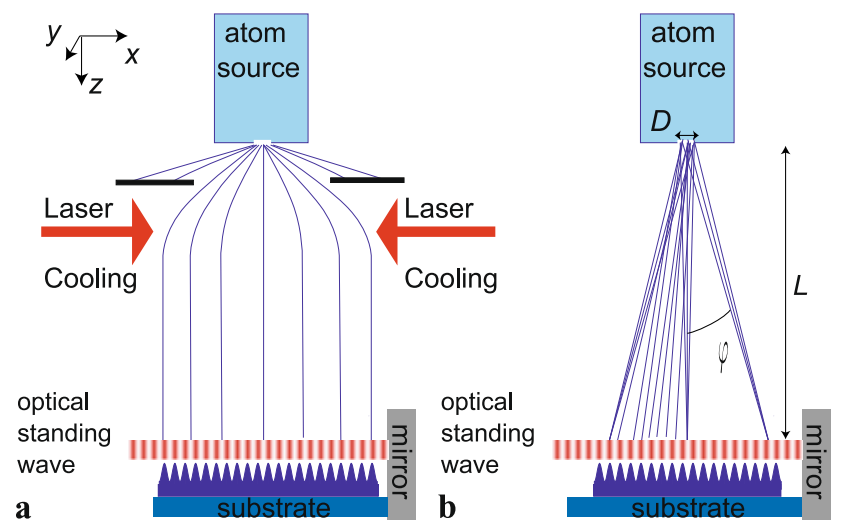

Fig. 1 Schematic overview of atomic nanofabrication using (a) laser cooling, creating a globally collimated atom beam, compared to (b) geometric cooling, creating a locally collimated atom beam. $D$ is the diameter of the opening of the atom source, $L$ is the atom source-sample distance, and $\varphi$ is the angle under which the atom beam locally impinges on the sample

to be small to enable sharp imaging. This can be achieved by geometric collimation, shown schematically in Fig. 1: an atom beam from a small beam source opening (e.g. $1 \mathrm{~mm}$ ) with a large source-sample distance (e.g. $1 \mathrm{~m}$ ) has a small local transverse velocity spread on the sample. In Fig. 1, we find a distinct difference between laser cooling and geometric collimation. A laser cooled beam aligns uniformly to the array of focussing lenses in the optical standing wave. However, with geometric collimation the atom beam aligns perfectly orthogonal to the focussing field at one point, but not over the entire standing wave.

In a setup without laser cooling, the transverse velocity distribution of the atom beam near the substrate is determined by the longitudinal velocity of the atom beam, the size of the atom beam source, the source-sample distance, and the position on the substrate. The longitudinal velocity distribution is that of an effusive beam, with average longitudinal velocity $\left\langle v_{\text {long }}\right\rangle$. At a point on the substrate the angular distribution of an atom beam emerging from a circular hole with diameter $D$ at a distance $L$ from the substrate, as shown in Fig. 1, is well approximated by a Gaussian angular distribution with a root-mean-square (RMS) width of $D /(4 L)$. The transverse velocity distribution thus has an RMS spread of $\sigma v_{\text {trans }}=(D /(4 L))\left\langle v_{\text {long }}\right\rangle$. The local average transverse velocity, $\left\langle v_{\text {trans }}\right\rangle$, is a function of position on the substrate. At each position where the beam impinges under an angle, $\varphi$, as seen in Fig. $1,\left\langle v_{\text {trans }}\right\rangle=\varphi\left\langle v_{\text {long }}\right\rangle$, as $\varphi$ is small in the experimental setup.

Experimentally, we have found atom lithographic structure formation of $\mathrm{Fe}$ over areas of up to $400 \mu \mathrm{m} \times 6 \mathrm{~mm}$ on a single substrate. An example of an atomic force microscopy (AFM) scan is shown in Fig. 2, where a $2 \times 2 \mu \mathrm{m}^{2}$ area is shown with 186-nm periodic line structures of 6-nm height. In our setup $L=0.74 \mathrm{~m}, D=1 \mathrm{~mm},\left\langle v_{\text {long }}\right\rangle=$
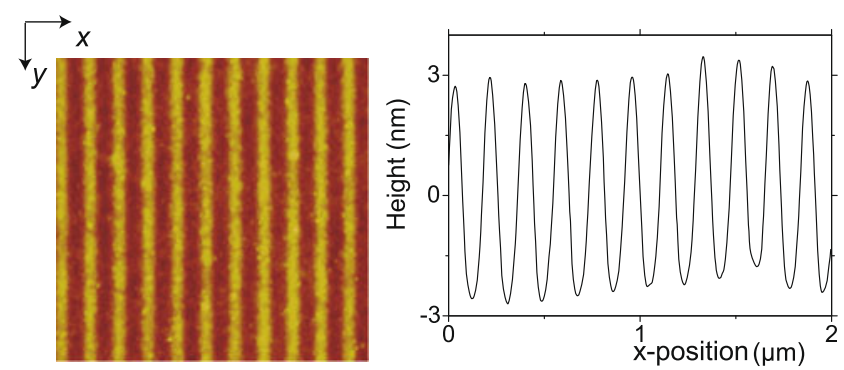

Fig. $22 \times 2 \mu \mathrm{m}^{2}$ AFM image (left) and average line cross section (right) of atom lithographic deposition of Fe without laser cooling

$1.0 \times 10^{3} \mathrm{~m} / \mathrm{s}$, and $\sigma v_{\text {trans }}=0.35 \mathrm{~m} / \mathrm{s}$. As the total size of the structured area in the $x$ direction (perpendicular to the lines) is up to 6-mm long, $\varphi$ varies over an $8 \mathrm{mrad}$ range, so that $\left\langle v_{\text {trans }}\right\rangle$ varies over an $8 \mathrm{~m} / \mathrm{s}$ range, more than an order of magnitude larger than $\sigma v_{\text {trans }}$.

In this article we investigate the effects of $\left\langle v_{\text {trans }}\right\rangle$. We show experimental results of structure height, width, and shape. We will also elaborate on two issues that influence structure formation on the surface: diffusion of atoms on the substrate after deposition and effects of scattering and interference of the standing wave field grazing over a substrate. When we take these diffusion and interference effects into account, we can understand structure formation in a geometrically collimated atom beam using a semiclassical Monte Carlo model.

Our paper is organised as follows: we first discuss a Monte Carlo model for atom lithographic focussing and present simulations using this model with transverse velocities included. Then we present the experimental setup, show the experimental results of structure formation, and compare them with simulations. We show how the effect of the light field configuration influences the experimental results. Furthermore, we show what the impact on the results is of diffusion of atomic depositions on the substrate. Finally, we discuss further possibilities and improvements of atom lithography without laser cooling and propose a novel scheme for a subwavelength patterning technique, based on geometrically collimated atom beams.

\section{Simulations}

The potential of an atom in a near-resonant light field follows from the so-called dressed-state model. The energies of dressed states $|1\rangle$ and $|2\rangle$ in a light field with a detuning $\Delta$ from resonance are [18]

$E_{1,2}=-\frac{\hbar \Delta}{2} \pm \frac{\hbar}{2} \sqrt{\Delta^{2}+\Gamma^{2} \frac{I(\mathbf{r})^{2}}{2 I_{\mathrm{s}}}}$.

Here $I(\mathbf{r})$ is the local intensity, $I_{\mathrm{S}}$ is the saturation intensity, and $\Gamma$ is the natural line width. 
The light field intensity in our simulation is modelled as a Gaussian standing wave (SW):

$I(\mathbf{r})=\frac{8 P}{\pi w^{2}} \sin ^{2}(k x) \cdot \exp \left(-2 \frac{z^{2}}{w^{2}}\right) \cdot \exp \left(-2 \frac{y^{2}}{w^{2}}\right)$.

Here $k=2 \pi / \lambda, w$ is the waist of the laser beam, $P$ is the laser power, and $x, y, z$ are positions as shown in Fig. 1 . In our experiments we use focussed Gaussian laser beams, but we disregard wavefront curvature because experiments are performed well within a Rayleigh length of the focus position.

In the experiment we use light fields with large positive detuning $\Delta \geq 50 \Gamma$, so that ground-state atoms entering the light field will initially be in the $|1\rangle$ dressed state adiabatically connected to the atomic ground state. This state has the lowest energy for the minimum intensity of the light and therefore atoms are focussed towards a local intensity minimum.

We use Fe atoms and a $\lambda=372 \mathrm{~nm}$ light field that is near resonant with the ${ }^{5} D_{4} \rightarrow{ }^{5} F_{5}$ transition in Fe. This transition is not fully closed, but as we use light relatively far detuned from resonance, optical pumping to other atomic states is ignored. Characteristics of the transition are given in Table 1.

To simulate the focussing process, we use a Monte Carlo trajectory calculation. Each simulation uses $10^{5}$ atoms which are considered as classical point particles moving in the dressed-state potentials. Initially all atoms are in the $|1\rangle$ dressed state at $z=-2 w$. The trajectories are calculated by numerically integrating the Newtonian equation of motion in three dimensions until the atoms reach $z=w / 2$. The atomic velocity distribution mimics that of our experimental setup in both longitudinal and transverse directions.

Spontaneous emission effects are included in our model, although these effects are not large for the large detunings and moderate intensity levels in our experiments. Direct heating by the random photon recoils from the spontaneous emissions ('spontaneous diffusion') is included, as well as 'stimulated diffusion' due to dressed state changing spontaneous emissions. After each spontaneous emission, a random photon recoil momentum is added to the atom's momentum and the possibility that the atom switches to the other dressed state is taken into account.

To include the effects of magnetic substates, the atoms are uniformly distributed over the magnetic substates and

Table 1 Characteristics for iron and the transition used

\begin{tabular}{lll}
\hline Transition & & ${ }^{5} D_{4} \rightarrow{ }^{5} F_{5}$ \\
Wavelength & $\lambda(\mathrm{nm})$ & 372.0 \\
Line width & $\Gamma / 2 \pi(\mathrm{MHz})$ & 2.58 \\
Saturation intensity & $I_{\mathrm{S}}\left(\mathrm{W} / \mathrm{m}^{2}\right)$ & 62 \\
Doppler velocity & $v_{\mathrm{d}}(\mathrm{m} / \mathrm{s})$ & 0.1 \\
\hline
\end{tabular}

the appropriate Clebsch-Gordan coefficients are assigned to each atom, assuming a linearly polarised light field. The local intensity is multiplied by the Clebsch-Gordan coefficient, resulting in different potentials and thus different focal lengths for each magnetic substate. The possibility that the magnetic substate of an atom changes during spontaneous emission is neglected.

\section{Simulation results}

In Fig. 3 we show some typical atomic trajectories of atoms moving from top to bottom through the SW potential of a light field with $\Delta=150(2 \pi) \mathrm{MHz}, P=20 \mathrm{~mW}$, and $w=$ $90 \mu \mathrm{m}$. Atoms are focussed towards the intensity minima at $x=0 \mathrm{~nm}$ and $x= \pm 0.186 \mu \mathrm{m}$, but the trajectories do not share a single focal point. This is partly due to the effect of the different potentials for the magnetic substates and the large chromatic abberation, as the RMS spread of $v_{\text {long }}$ is $357 \mathrm{~m} / \mathrm{s}$.

In our experiments the substrate is positioned at the point of maximum laser intensity, $z=0 \mu \mathrm{m}$; therefore, we take the simulated atomic distribution in that plane. On the left of Fig. 4 we show atomic distributions at $z=0 \mu \mathrm{m}$ and around $x=0 \mu \mathrm{m}$ (thus $\varphi=0 \mathrm{mrad}$ ) for a light field of $w=90 \mu \mathrm{m}$ at several $y$ positions. We observe that for non-zero $y$ positions, where local light intensity is lower, atoms are focussed less tightly resulting in lower and broader distributions. To characterise the simulation results, we fit the atomic distribution with a Gaussian; an example is shown in the righthand panel of Fig. 4. The values we find in the Gaussian fit for the peak height over the pedestal, $h$, and the full width at half maximum, $w_{h}$, can be compared to experimental results. To convert simulated atomic distributions into height values, we take into account the Fe flux in our experiment and the fraction of atoms that are in the atomic ground state and therefore interact with the potential.

In the left-hand panel of Fig. 5 we show simulation results for focussing by the same light field at $y=0 \mu \mathrm{m}$, but

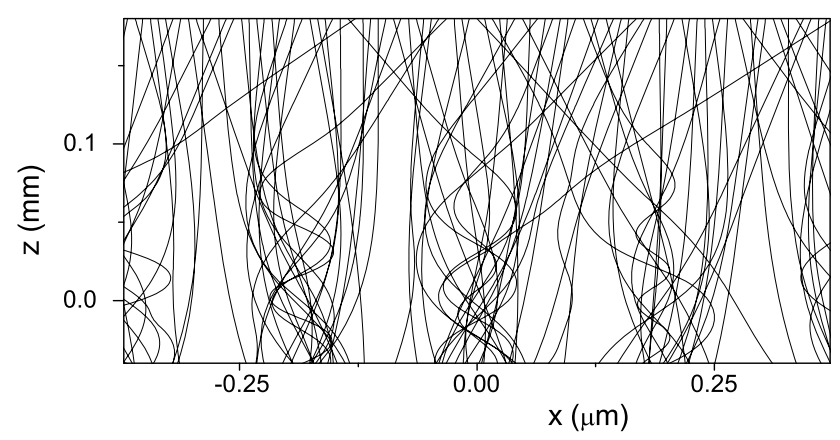

Fig. 3 Overview of atomic trajectories in a SW light field of $P=20 \mathrm{~mW}, w=90 \mu \mathrm{m}$, and $\Delta=150(2 \pi) \mathrm{MHz}$ 
Fig. 4 Simulated atomic distribution of atoms around $x=0 \mu \mathrm{m}$ (thus $\varphi \simeq 0 \mathrm{mrad}$ ) in the plane $z=0 \mu \mathrm{m}$ for atoms focussed by a SW light field with $w_{0}=90 \mu \mathrm{m}, P=20 \mathrm{~mW}$. Left: at positions in the light field $y=0 \mu \mathrm{m}$ (thick black curve), $y=45 \mu \mathrm{m}$ (dotted red), $y=90 \mu \mathrm{m}$ (dashed blue). Right: Gaussian fit of $y=0 \mu \mathrm{m}$ (thin red line), indicating peak height $h$ and full width at half maximum $w_{h}$
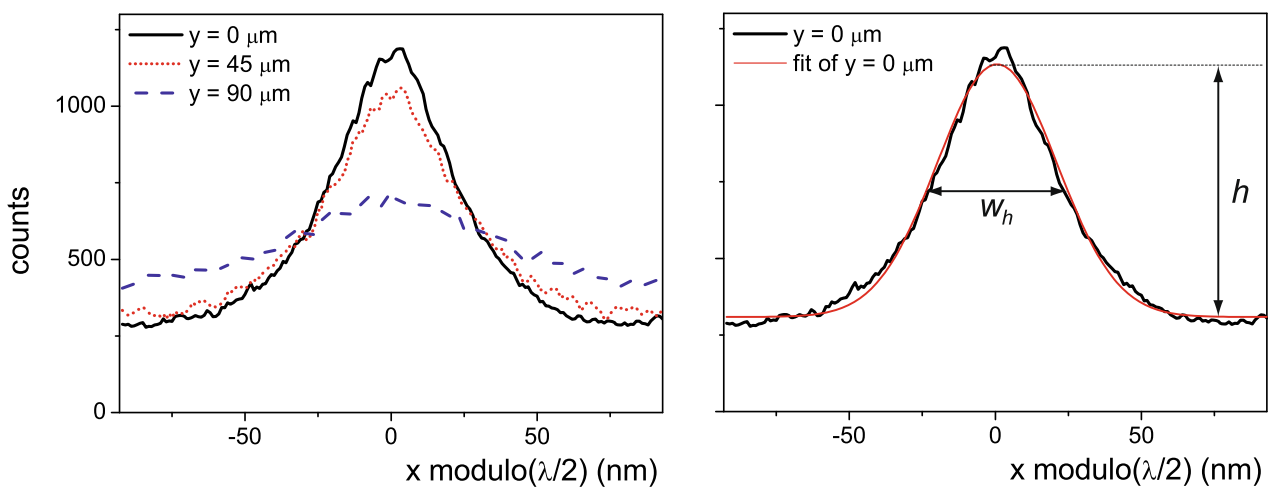
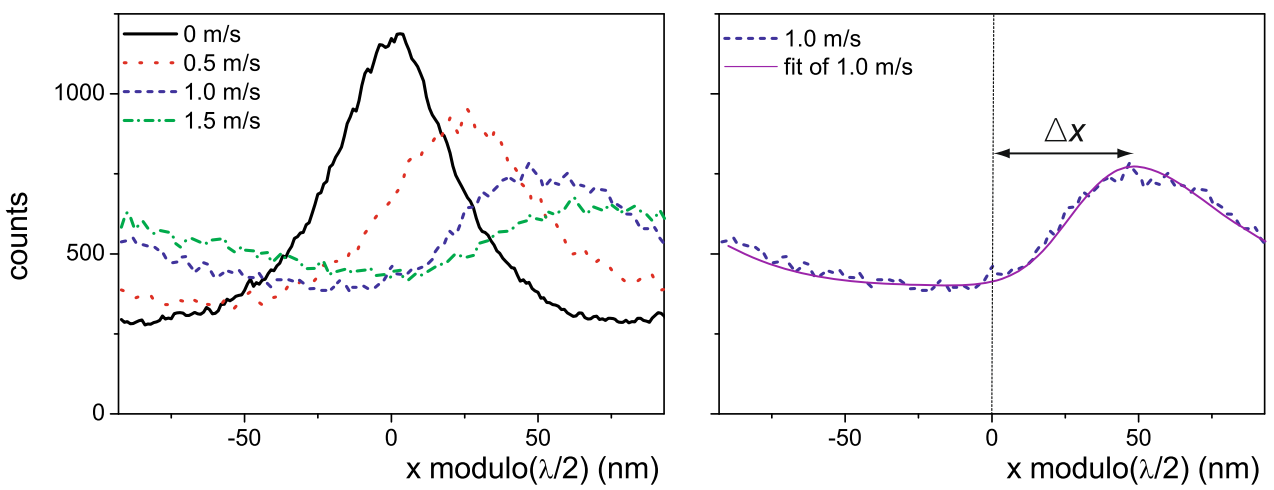

Fig. 5 Left: simulated atomic distribution of atoms in the plane $z=0 \mu \mathrm{m}$ and $y=0 \mu \mathrm{m}$ for atom beams with $\left\langle v_{\text {trans }}\right\rangle$ of $0 \mathrm{~m} / \mathrm{s}$ (solid black curve), $0.5 \mathrm{~m} / \mathrm{s}$ (dotted red), $1.0 \mathrm{~m} / \mathrm{s}$ (dashed blue), and $1.5 \mathrm{~m} / \mathrm{s}$ (dash-dotted green), focussed by a SW light field with $w_{0}=90 \mu \mathrm{m}$ and $P=20 \mathrm{~mW}$. Note that $\left\langle v_{\text {trans }}\right\rangle=1 \mathrm{~m} / \mathrm{s}$ is equal to $\varphi=1 \mathrm{mrad}$. Right: simulated atomic distribution of $\left\langle v_{\text {trans }}\right\rangle=1 \mathrm{~m} / \mathrm{s}$, fitted with a skewed Gaussian and indicating peak shift $\Delta x$

cal length of the lens, resulting in e.g. $f=66 \mu \mathrm{m}$ for $w=150 \mu \mathrm{m}$ and $P=55 \mathrm{~mW}$. It should be noted that the shift of the peak positions will be nearly impossible to observe experimentally, as the change in distance between two neighbouring structures will be on the order of $10^{-11} \mathrm{~m}$.

For larger $\varphi, \Delta x$ moves towards $\lambda / 4=93 \mathrm{~nm}$, the position where the SW potential is maximal. If atoms move beyond $\Delta x=\lambda / 4$, they move through the SW potential maximum towards a neighbouring potential minimum. In that case an atom is no longer focussed by a single lens, but is moving through a periodic potential. Atoms are then more likely to be near the maxima of the potential where their kinetic energy and thus speed is lower. At higher values of $\varphi$, the peak position therefore remains near the maximum of the potential and thus at $\Delta x=\lambda / 4=93 \mathrm{~nm}$.

A second effect of non-zero $\left\langle v_{\text {trans }}\right\rangle$ was observed in the skewness of the lines. In the right-hand panel of Fig. 6 we show the skewness parameter $\alpha$ of line structures modelled for a SW potential of $w=90 \mu \mathrm{m}$ and $P=20 \mathrm{~mW}$; other standing waves give similar results. We find that for small $\varphi$, $\alpha$ increases linearly with $\varphi$ up to $\varphi=1.5 \mathrm{mrad}$. At this angle where we showed on the left of Fig. 6 that $\Delta x$ changes from a linear regime to the $\Delta x=\lambda / 4$ level, the skewness also 
Fig. 6 Effects of non-zero $\varphi$ on simulated atomic distributions.

Left: $\Delta x$ vs. $\varphi$ for light fields of $w=150 \mu \mathrm{m}$ and $P=55 \mathrm{~mW}$

(blue triangle); $w=90 \mu \mathrm{m}$ and

$P=20 \mathrm{~mW}$ (red dots);

$w=60 \mu \mathrm{m}$ and $P=50 \mathrm{~mW}$

(black squares), indicating

$\Delta x=\lambda / 4=93 \mathrm{~nm}$ (dashed

line). Right: skewness parameter

$\alpha$ vs $\varphi$ of the deposited

structures for $w=90 \mu \mathrm{m}$ and

$P=20 \mathrm{~mW}$
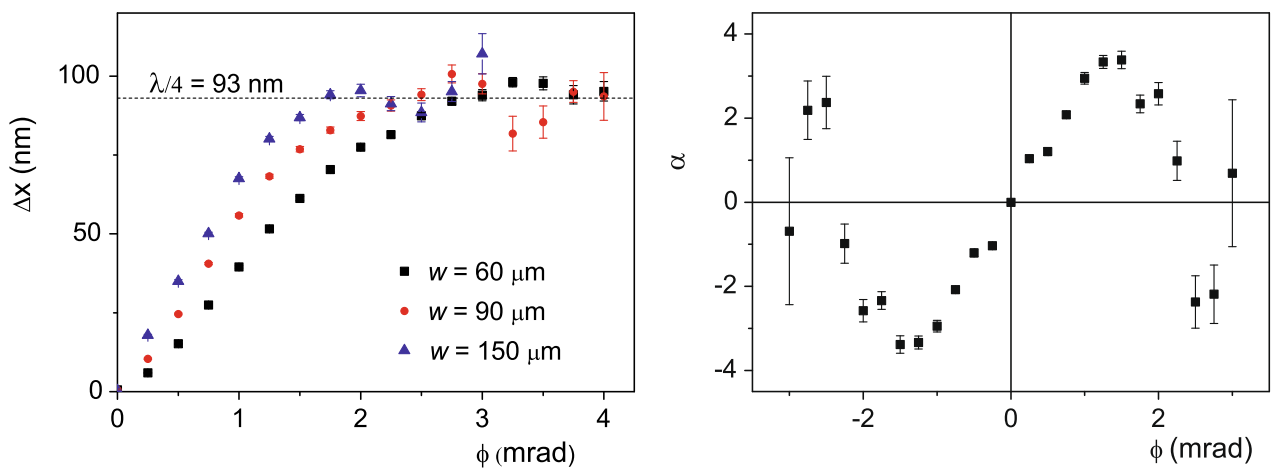

Table 2 Characteristics of the atom beam

\begin{tabular}{lll}
\hline Atom source diameter & $D(\mathrm{~mm})$ & 1 \\
Atom source-sample distance & $L(\mathrm{~m})$ & 0.74 \\
Atom source temperature & $T(\mathrm{~K})$ & 2000 \\
Average longitudinal velocity & $\left\langle v_{\text {long }}\right\rangle(\mathrm{m} / \mathrm{s})$ & $1.0 \times 10^{3}$ \\
Longitudinal velocity spread (RMS) & $\sigma v_{\text {long }}(\mathrm{m} / \mathrm{s})$ & 357 \\
Transverse velocity spread (RMS) & $\sigma v_{\text {trans }}(\mathrm{m} / \mathrm{s})$ & 0.35 \\
\hline
\end{tabular}

changes behaviour. For larger values of $\varphi$, the skewness is ill determined and fluctuating around $\alpha=0$.

\section{Experimental setup}

A schematic of our setup is shown on the right-hand side of Fig. 1. To be able to deposit Fe at a reasonable flux, we require temperatures up to $2000 \mathrm{~K}$, for which we use a homebuilt effusive Knudsen cell [19]. This Knudsen cell has a circular opening of 1-mm diameter and is located $740 \mathrm{~mm}$ from the sample. The characteristics of the atom beam and the beam geometry are given in Table 2 . The thermal population of $\mathrm{Fe}$ atoms at $2000 \mathrm{~K}$ gives an occupation of the ground state of $47 \%$, which is the only state interacting with the light field. Substrates are exposed to the atom beam for two hours, resulting in deposited Fe films of an average thickness of approximately $15 \mathrm{~nm}$.

The 372-nm light needed to excite the ${ }^{5} D_{4} \rightarrow{ }^{5} F_{5}$ transition of $\mathrm{Fe}$ is produced by a titanium-sapphire laser, frequency doubled with a LBO crystal in a ring cavity and frequency locked using the method described in [20]. An acousto-optical modulator is used to introduce a detuning $\Delta=150(2 \pi) \mathrm{MHz}$. The 372-nm light is focussed onto a mirror in the vacuum to create a standing wave with waist size between 60 and $150 \mu \mathrm{m}$. The retroreflected beam is aligned to the incoming beam to less than 1-mrad deviation. The substrate is positioned in the standing wave as shown top right in Fig. 7, such that the power in the retroreflected beam is $50 \%$ of the maximum power reflected off the mirror.

In Fig. 7 we show the sample holder, on which the samples are clamped and a mirror is mounted. Substrates are sil-

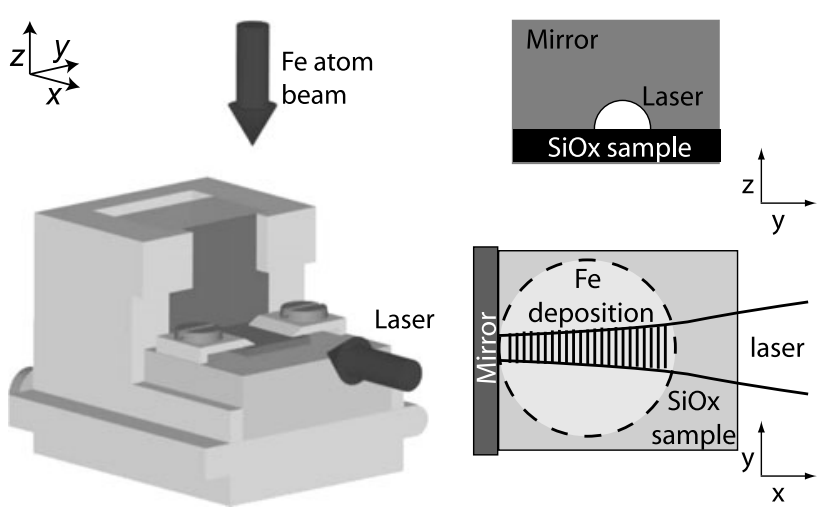

Fig. 7 Left: sample holder. Top right: view along laser field showing $50 \%$ cut-off. Bottom right: view along Fe atom beam showing overlap of laser and deposition area on sample where line structures are created

icon oxide $(\mathrm{SiOx})$, which is amorphous to prevent ordered growth effects due to crystalline substrates. The mirror can be aligned perpendicular to the substrate within $1 \mathrm{mrad}$ ex vacuo. The sample holder is placed in the deposition chamber, which has a typical pressure below $10^{-8}$ mbar. In the deposition chamber, the atom beam has a diameter of $7 \mathrm{~mm}$ and it impinges normal to the substrate near the centre of the substrate. In the bottom right of Fig. 7 we show that line structures can be created where the laser field overlaps the area of Fe deposition.

The resulting structures have been imaged using atomic force microscopy (AFM). We measured under ambient conditions on a Digital Instruments Dimension 3100 AFM in tapping mode, using Si cantilevers with a radius of curvature less than $10 \mathrm{~nm}$. As our structures have typical ratios of $h / w_{h}$ below $10^{-1}$, we disregard tip artefacts. Measuring along the $y$ direction gives information of the influence of the local laser intensity, where we take $y=0$ at the position of maximum laser intensity. Measuring along the $x$ direction gives insight into the effects of the transverse velocity of the Fe atom beam, where $x=0$ corresponds to $\left\langle v_{\text {trans }}\right\rangle=0$. AFM measurements are performed on areas of $250 \mathrm{~nm}$ along the lines and $2 \mu \mathrm{m}$ perpendicular to the lines, so that 11 lines are visible. The image is averaged over the $y$ direction and in the resulting cross section of the line structures each line 
Fig. $8 h$ (left) and $w_{h}$ (right) of simulated (red dots) and experimentally measured lines (black squares) for different $y$ positions and different laser field settings, from top to bottom $w=60 \mu \mathrm{m}$ and $P=50 \mathrm{~mW} ; w=90 \mu \mathrm{m}$ and $P=20 \mathrm{~mW} ; w=150 \mu \mathrm{m}$ and $P=55 \mathrm{~mW}$. A normalised laser intensity profile is shown in the left-hand images (grey area)
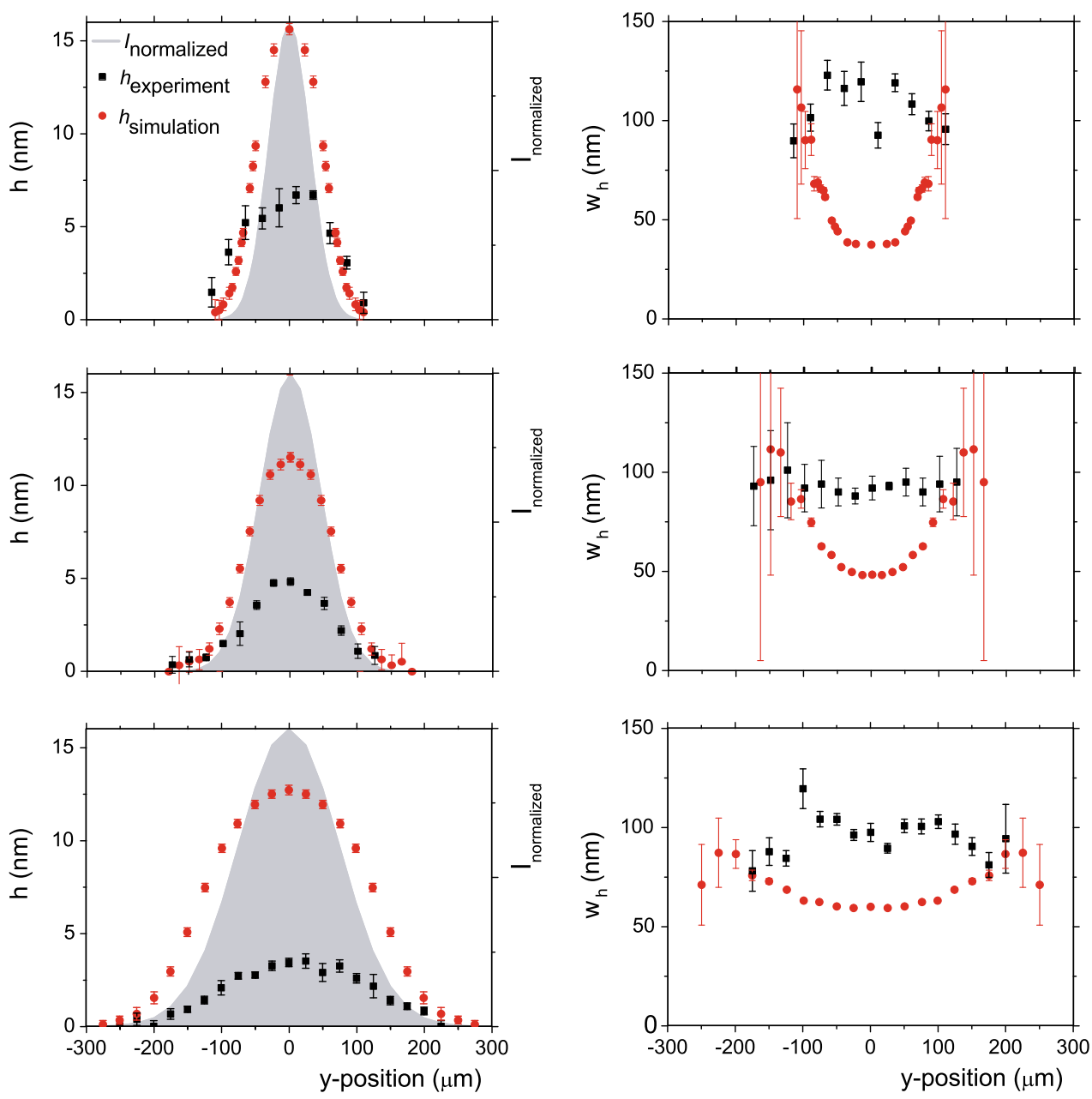

is fitted with a Gaussian giving average and spread values of $h$ and $w_{h}$.

\section{Experimental results}

In Fig. 8 we present experimental and simulation results for $h$ and $w_{h}$ of deposited lines as a function of $y$ position, when $x=0$ (thus $\left\langle v_{\text {trans }}\right\rangle=0$ ). Three settings of $P$ and $w$ are shown. In Fig. 9 we present experimental and simulation results of lines deposited at $y=0 \mathrm{~mm}$ and thus at the maximum intensity level of the laser field as a function of $x$ position, which is proportional to $\left\langle v_{\text {trans }}\right\rangle$. In our simulations the highest lines are found for the highest laser power and for an atom beam with $\left\langle v_{\text {trans }}\right\rangle=0$. This behaviour is confirmed in our experiments.

In Fig. 8 we find that structure formation is influenced by the local intensity of the laser field. The simulation results show a bell-shaped dependence of $h$ on $y$ position, which is wider and more flat topped than the intensity profile. This indicates that the focussing is less sensitive to intensity changes at high intensity. This is confirmed by the simulation values of $w_{h}$, which are reasonably constant for $|y| \lesssim w$, while, for larger $y$, corresponding to lower intensities, $w_{h}$ increases. The experimental results for $h$ match the simulations for the outside positions, but differ greatly for $|y| \lesssim w$. At $y=0, h$ is two to three times smaller than expected. Experimental values of $w_{h}$ hardly depend on $y$ position. Values of $w_{h}$ are between 80 and $120 \mathrm{~nm}$, which corresponds to simulation values at the extreme $y$ positions, but simulation values of $w_{h}$ around $y=0$ are significantly smaller, down to $35 \mathrm{~nm}$.

The influence of local $\left\langle v_{\text {trans }}\right\rangle$ on structure formation is shown in Fig. 9. Experimentally, we observe structures even for large values of $x>4 \mathrm{~mm}$, corresponding to $\left\langle v_{\text {trans }}\right\rangle=$ $6 \mathrm{~m} / \mathrm{s}$. This is more than an order of magnitude larger than $\sigma v_{\text {trans }}$ and nearly two orders of magnitude larger than the transverse velocity associated with Doppler cooled beams, where $\sigma v_{\text {trans }}=v_{\mathrm{d}}=0.1 \mathrm{~m} / \mathrm{s}$ (Table 2). At those values for $\left\langle v_{\text {trans }}\right\rangle$, atoms in the atom beam can pass through a maximum in the focussing potential and are therefore no longer restricted to interact with a single lens.

The simulation results in Fig. 9 indicate that the highest and narrowest lines are deposited for $x=0 \mathrm{~mm}$, where 
Fig. $9 h$ (left) and $w_{h}$ (right) of simulated (red dots) and experimentally measured lines (black squares) for different $x$ positions (thus different $\left\langle v_{\text {trans }}\right\rangle$ ) and different laser field settings, from top to bottom $w=60 \mu \mathrm{m}$ and $P=50 \mathrm{~mW} ; w=90 \mu \mathrm{m}$ and $P=20 \mathrm{~mW} ; w=150 \mu \mathrm{m}$ and $P=55 \mathrm{~mW}$
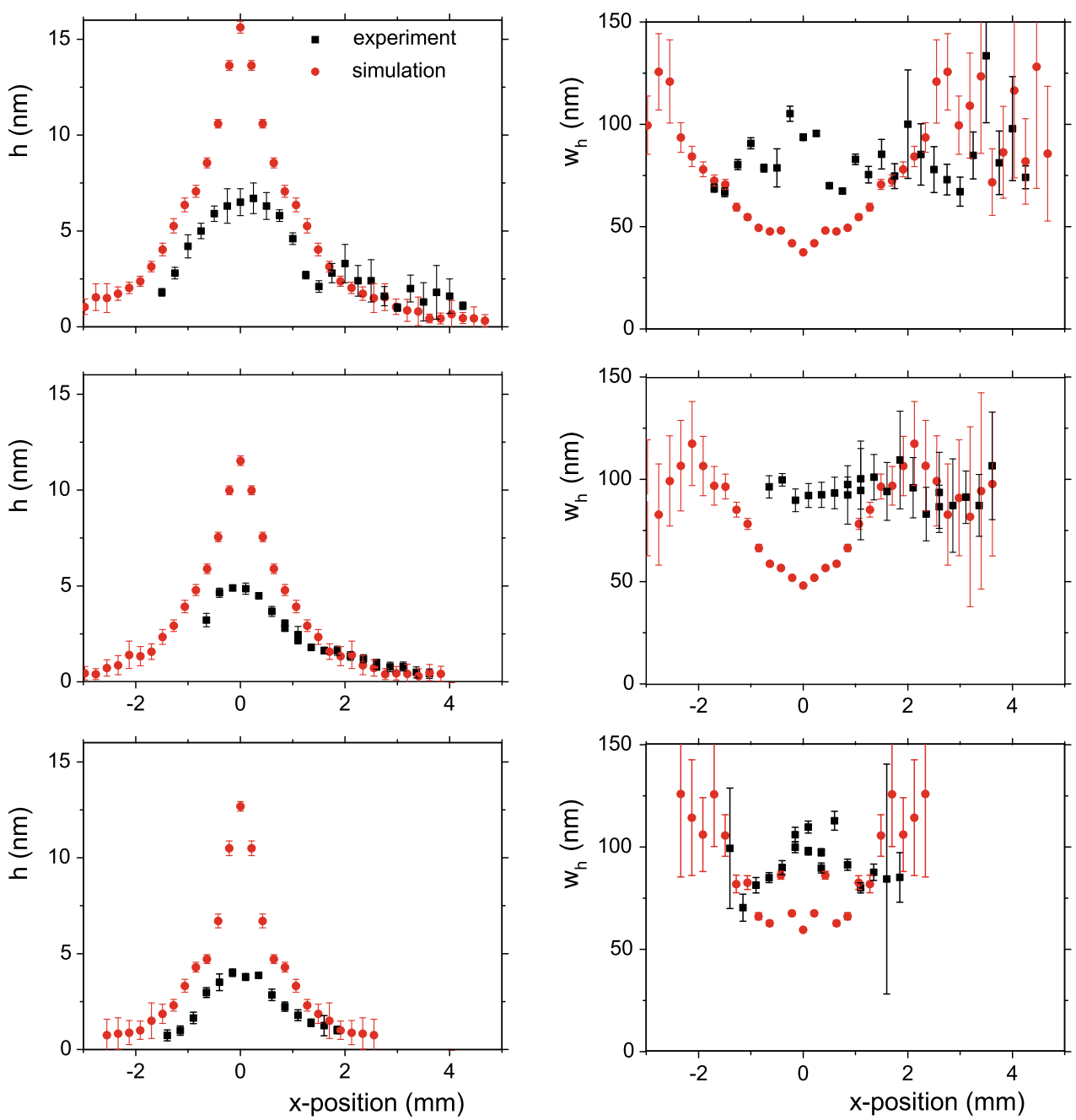

$\left\langle v_{\text {trans }}\right\rangle=0 \mathrm{~m} / \mathrm{s}$. These results show a sharp decrease in line height for non-zero $\left\langle v_{\text {trans }}\right\rangle$. Experimental results indeed have a maximum for $h$ at $x=0 \mathrm{~mm}$, but this peak is less pronounced than in the simulations. The measured widths, $w_{h}$, are all between 80 and $120 \mathrm{~nm}$, well over the simulated values around $x=0 \mathrm{~mm}$ but comparable at the extreme values of $x$. Both Figs. 8 and 9 indicate that the simulations predict broad, low structures correctly, but fail in the case of optimal focussing, with narrow and high lines. This points towards the presence of an additional broadening mechanism not accounted for in the simulations.

This notion is confirmed by a comparison of the simulated and experimental values for the product of $h$ and $w_{h}$, indicating the total amount of focussed material. In Fig. 10 we show the product for one field setting; results are similar for other settings. We find that the simulated product of $h$ and $w_{h}$ of the focussed lines compares much better to the experimental results, with errors of only about $20 \%$.

In atom lithography, broadening of structures has been found previously $[21,22]$. Two explanations have been proposed: the distortion of the light field by the presence of the substrate and the mobility of deposited atoms on the surface.

The effect of the substrate on the light field has been modelled by Anderson et al. [21]. Following their approach, we calculated the light field near the centre of our substrate assuming that the surface and mirror are fully reflecting, from an incoming Gaussian laser field cut off at maximum intensity by the substrate. We find a dark zone just above the surface of about 10- $\mu \mathrm{m}$ height, while the intensity is nearly two times higher than expected at about $20 \mu \mathrm{m}$ from the surface. Further away from the surface, the intensity patterns is more similar to the incoming Gaussian pattern. The assumption that the substrate and the mirror are fully reflective is an extreme; our experiment is much more difficult to model as Fe coats our $\mathrm{SiOx}$ substrate during the experiment, so that reflection coefficients and associated phase terms change during the experiment.

The dark area just above the surface can allow for defocussing of the atom beam. In our simulations, we find that $\sigma v_{\text {trans }}$ in the plane $z=0$ is $0.53 \mathrm{~m} / \mathrm{s}$ for the strongest focussed beams, leading to a maximal broadening of $6 \mathrm{~nm}$ on 
Fig. 10 Measured (black squares) and simulated (red circles) product of $h$ and $w_{h}$ for light field of $w=90 \mu \mathrm{m}$, $P=20 \mathrm{~mW}$ as a function of $y$ position (left) and $x$ position (right)
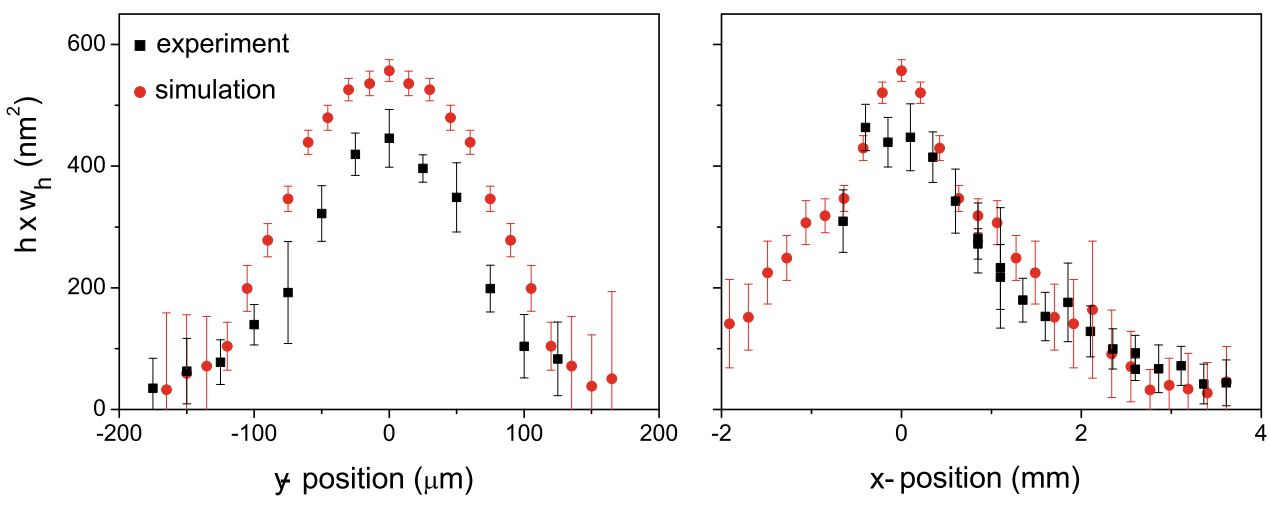

Fig. 11 Cross sections of nanolines created by depositing Fe through physical masks on $\mathrm{SiOx}$ (left) and $\mathrm{Si}\langle 111\rangle$ (right). Dotted red curve: geometric expected structures from the 80-nm-wide mask openings. Solid black curve: measured AFM results. Dashed blue curve: a model convoluting the geometric expectation with a Gaussian of $\sigma=23 \mathrm{~nm}$ (left) and $\sigma=12 \mathrm{~nm}$ (right)

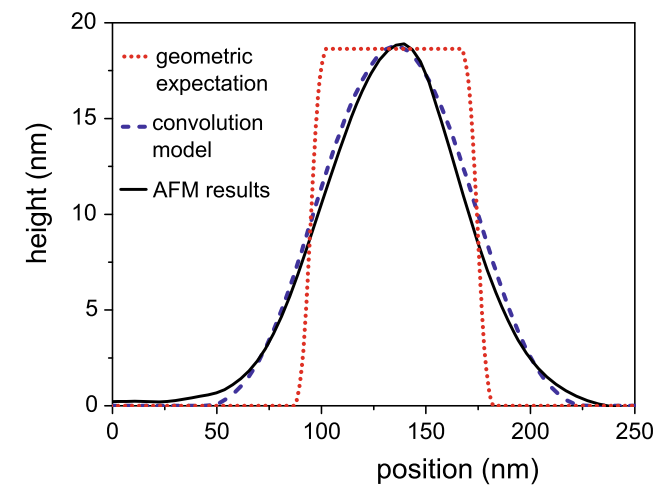

both edges of the focussed line structure in a 10- $\mu \mathrm{m}$ high dark area. As the intensity is higher just above the dark zone over the substrate, the focussing strength might be greater at some points, thus also leading to stronger defocussing. However, it seems that the divergence in the dark zone over the substrate alone cannot account for the broadening of the structures from $35 \mathrm{~nm}$ to over $80 \mathrm{~nm}$ that we observe in Figs. 8 and 9.

The mobility of atoms on a surface and the resulting blurring of structures have also been a subject of study in the field of atom lithography. Ballistic deposition models [23], terrace and step-edge diffusion [24], and polluted adatom limited diffusion [22] have been proposed. The latter is consistent with the experimental data of [21], but there is no complete understanding of atom mobility on the substrate. We have observed very clear effects in our setup while depositing structures on $\mathrm{SiOx}$ and $\mathrm{Si}\langle 111\rangle$. Previously reported Fe structures on $\mathrm{Si}\langle 111\rangle$ with native oxide created with a very similar setup had a typical $w_{h}$ of $50 \mathrm{~nm}$ [17], while our current line structures deposited on $\mathrm{SiOx}$ have a typical $w_{h}$ of over $80 \mathrm{~nm}$.

As a reference experiment to the atom lithographic experiments on $\mathrm{Si}\langle 111\rangle$ and $\mathrm{SiOx}$, we have deposited Fe through physical masks with line patterns, to deposit well-defined structures onto $\mathrm{Si}\langle 111\rangle$ or $\mathrm{SiOx}$ substrates. The masks are made of 100-nm-thick silicon nitride which are e-beam patterned with line patterns $80 \pm 5$-nm wide and several hundred $\mu \mathrm{m}$ long. The masks are clamped directly on the substrate, so that the mask-substrate distance is less than $10 \mu \mathrm{m}$. We have deposited 15- to 20-nm-thick layers of Fe through these line masks and measured their geometry with AFM. In Fig. 11 we show cross sections of Fe nanolines deposited on $\mathrm{SiOx}$ and $\mathrm{Si}\langle 111\rangle$. We find that there is a significant influence of the substrate. This is known in the literature for other materials, e.g. Tun et al. [25] observed very different structure formation of gold deposited on various substrates through physical masks.

In Fig. 11 we have included the expected deposition profile based on the geometry of the mask, the divergence of the atom beam, and the mask-substrate distance and find that this does not match the experimental results. We model the substrate influence as a convolution of the expected profile with a Gaussian function, of which the standard deviation $\sigma$ gives a measure for diffusion. In Fig. 11 we show that this gives a good match to measured profiles for $\sigma=12 \mathrm{~nm}$ for $\mathrm{Si}\langle 111\rangle$ and $\sigma=23 \mathrm{~nm}$ for $\mathrm{SiOx}$.

The value of $\sigma$ is not easily translated into an increase in width of structures in atom lithography. It should be noted that the full width at half height in Fig. 11 of the expected deposition profile, the model, and both measurements are nearly equal. As atom lithography creates Fe nanostructures on a Fe background, surface diffusion and broadening effects will be different from the freestanding structures shown in Fig. 11. However, the results indicate that diffusion effects of $\mathrm{Fe}$ on $\mathrm{SiOx}$ and $\mathrm{Si}\langle 111\rangle$ have a range of tens of $\mathrm{nm}$ and we find that the value $\sigma$ for $\mathrm{SiOx}$ is approximately twice 
Fig. 12 Overview of simulated (left) and measured (right) values of skewness $\alpha$ of nanostructures deposited with a light field of $w=150 \mu \mathrm{m}$ and $P=55 \mathrm{~mW}$
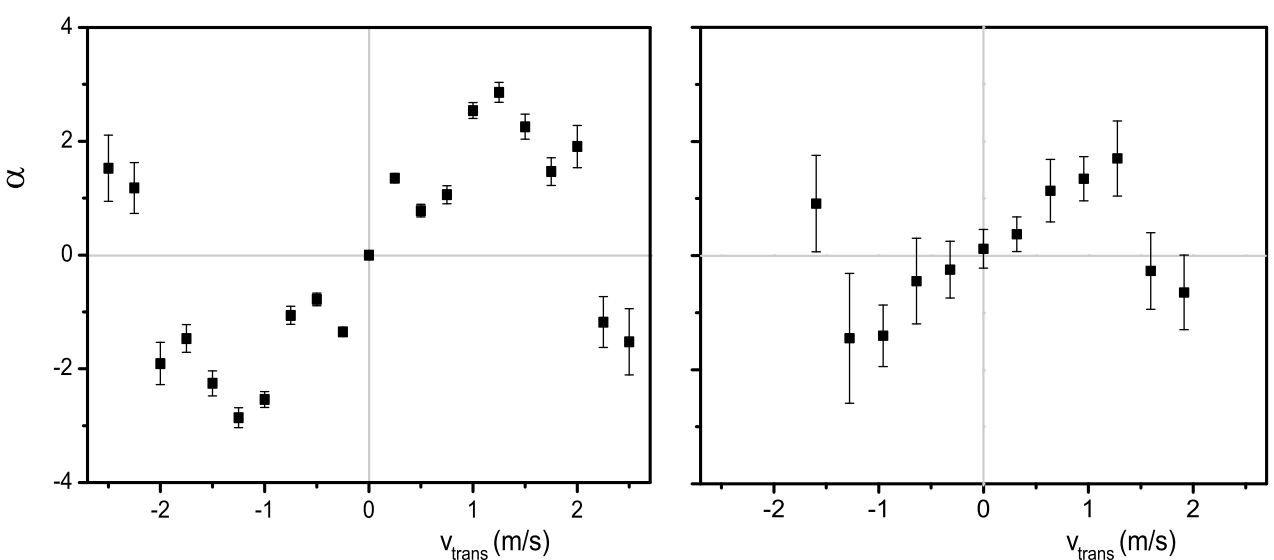

the value for $\mathrm{Si}\langle 111\rangle$. This matches well with the length scale over which the values of $w_{h}$ of atom lithographic lines differ in simulations ( $w_{h} \geq 35 \mathrm{~nm}$ ) and experimental results on $\mathrm{Si}\langle 111\rangle\left(w_{h} \gtrsim 50 \mathrm{~nm}\right)[17]$ and $\mathrm{SiOx}\left(w_{h} \gtrsim 80 \mathrm{~nm}\right)$.

Although it is likely that diffusion and broadening affect narrow and high structures more than broad structures, these results do not indicate how diffusion and broadening affect structure height and width for different deposited flux distributions. However, we have shown that substrate-dependent surface diffusion effects can contribute significantly to the observed differences in $w_{h}$ of our Monte Carlo model and the atom lithographic experiments.

The experiments show most of the effects found in the simulations qualitatively, although lines are broadened. We have therefore also investigated the skewness of our deposited structures, which we found in our simulations to be dependent on $\left\langle v_{\text {trans }}\right\rangle$. We show the skewness parameter for both simulated and experimental results in Fig. 12 for structures deposited with a light field of $w=150 \mu \mathrm{m}$ and $P=55 \mathrm{~mW}$. We clearly retrieve the linear trend for values of $\left\langle v_{\text {trans }}\right\rangle$ from -1.25 to $1.25 \mathrm{~m} / \mathrm{s}$ and for larger values of $\left\langle v_{\text {trans }}\right\rangle$ both simulation and experimental values break with the linear trend. Quantitatively, the measured values of $\alpha$ are smaller than the simulation results, which is to be expected as the observed broadening of the structures in experiments will decrease skewness.

\section{Discussion and conclusions}

In this article we have shown that atom lithography is possible with atom beams that have a local $\left\langle v_{\text {trans }}\right\rangle$ of up to a few $\mathrm{m} / \mathrm{s}$. This local $\left\langle v_{\text {trans }}\right\rangle$, which corresponds to an offset angle of the atom beam with respect to the SW, influences height, width, and shape of the deposited structures. These effects have been simulated and have been qualitatively observed in our samples. However, the observed structures are broader and lower than in the simulations. We find that this discrepancy can be partly explained by light scattering and interference near the reflective substrate. However, we find that our results are mostly influenced by a substratedependent diffusion mechanism. Whereas the simulation resulted in structure sizes of $w_{h} \geq 35 \mathrm{~nm}$, we do not find structures of $w_{h}<80 \mathrm{~nm}$ on SiOx substrates. In our previous experiments on $\mathrm{Si}\langle 111\rangle$ substrates we found smaller structures of $w_{h} \geq 50 \mathrm{~nm}$ [17]. A better understanding of surface effects and choice of substrate could therefore allow for better defined structures.

As our current structuring technique does not need laser cooling, we are no longer limited to atomic species that have closed optical transitions. This allows for the use of technologically interesting elements such as Ga, In, and Si [16], where laser cooling was shown to be experimentally challenging. We calculated the achievable flux densities without laser cooling for some elements of groups III and IV (Al, Ga, In, Si, Ge, and $\mathrm{Sn}$ ) in our current setup and atom beam source geometry using data from [27]. In all cases the deposition rate is at least $10 \mathrm{~nm}$ per hour at beam source temperatures lower than $2000 \mathrm{~K}$, which we use for Fe. As in our Fe experiment, the thermal occupation of any single (hyperfine) level of these atomic beams is significantly less than 1. Therefore, for example, optical pumping might be required to achieve good contrast in the atom lithographic focussing.

Atom lithography without laser cooling could furthermore be improved by changing the geometry of the setup and the atom beam source. We currently use a Knudsen cell with a round opening as an atom beam source, the size of which determines the local $\sigma v_{\text {trans }}$ on the sample. However, for line structures, only $\sigma v_{\text {trans }}$ perpendicular to the lines needs to be controlled and we can thus use slit-like source openings, with a large surface area, but a narrower size perpendicular to the lines. This allows us to increase flux with lower $\sigma v_{\text {trans. }}$. The source-substrate distance $L$ also influences $\sigma v_{\text {trans }}$. As $\sigma v_{\text {trans }}$ is inversely proportional to $L$, increasing $L$ can create locally better collimated beams. However, the flux is inversely proportional to $L^{2}$, which restricts 

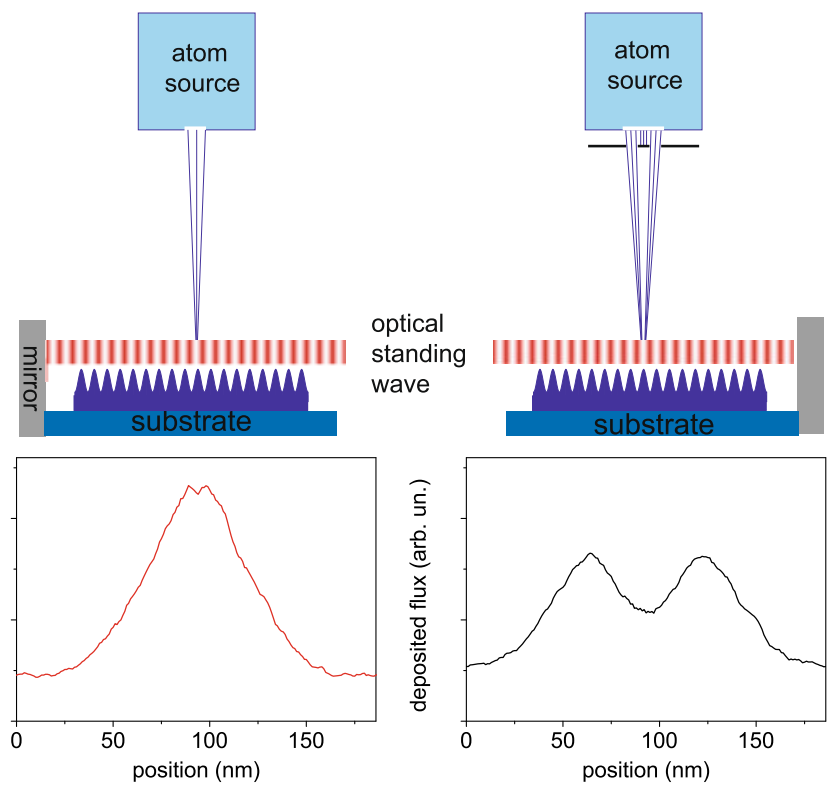

Fig. 13 Example of atom lithography using a patterned atom beam. Left top: atom beam impinging on a single SW lens from a 1-mm round source opening; Right top: atom beam impinging on a single SW lens from two $0.33-\mathrm{mm}$ slits positioned $0.75-\mathrm{mm}$ apart. Bottom: simulated atomic distribution after focussing of atom beams by a SW light field of $w=150 \mu \mathrm{m}$ and $P=55 \mathrm{~mW}$

large increases of $L$. A second advantage of a larger $L$ is that the offset angles $\varphi$ will be smaller, so that deposited structures are uniform over larger areas. Adjustment of the geometry of the setup and atom beam source can thus allow for faster deposition rates, lower $\sigma v_{\text {trans }}$, and/or a smaller influence of $\left\langle v_{\text {trans }}\right\rangle$.

Finally, we propose to use atom lithography without laser cooling to image patterns in the atomic beam source onto a substrate, similar to imaging in classical optics. We show the concept in Fig. 13. At the left an atom beam emerging from a single $D=1 \mathrm{~mm}$ round opening, at distance $L=0.74 \mathrm{~m}$, is focussed by a SW lens of $w=150 \mu \mathrm{m}$ and $P=55 \mathrm{~mW}$ as in our current setup. The magnification ratio is $f / L=8.9 \times 10^{-5}$, with $f=66 \mu \mathrm{m}$ the effective focus length we obtained from Fig. 6 . We find that this is similar to the reduction in size of the beam source opening $w_{h} / D=6.1 \times 10^{-5}$. The SW lens thus creates an image of the beam source onto the substrate. With laser cooled atom beams, this is impossible as laser cooling removes information of the beam source. We also simulated the focussed atomic distribution of an atom beam from a beam source in front of which we place two 0.33 -mm-wide slits spaced $0.75-\mathrm{mm}$ apart, as shown on the right of Fig. 13. The double slit is focussed to a double line pattern spaced 60-nm apart, reducing the size of the spacing by a factor $8 \times 10^{-5}$, again very similar to $f / L$. Using atom lithography without laser cooling to image structured beam sources allows for the creation of patterns which are repetitive over $\lambda / 2$ in a massively parallel way.
Considering the typical $w_{h}=80 \mathrm{~nm}$ of Fe on SiOx, our setup is not very suitable for this type of experiment, as multiple features might not be discernable on an 186-nm interval. However, in atom lithography structures have been observed with sizes down to $13 \mathrm{~nm}$ in sodium [26] and down to $29 \mathrm{~nm}$ in chromium [21], allowing for more intricate patterns within the corresponding lens sizes of $\lambda_{\mathrm{Na}} / 2=295 \mathrm{~nm}$ and $\lambda_{\mathrm{Cr}} / 2=213 \mathrm{~nm}$. More complex patterns could be possible if two perpendicular standing waves are used, creating a two-dimensional array of lenses, which would allow for two-dimensional patterning. This scheme will of course suffer from the large aberrations that optical lenses for atoms have. Nevertheless, potential applications in the fields of, for example, nanoengineering and nanomagnetism could make this scheme technologically interesting.

Acknowledgements This work is part of the research programme of the Foundation for Fundamental Research on Matter (FOM), which is part of the Netherlands Organisation for Scientific Research (NWO).

Open Access This article is distributed under the terms of the Creative Commons Attribution Noncommercial License which permits any noncommercial use, distribution, and reproduction in any medium, provided the original author(s) and source are credited.

\section{References}

1. G. Timp, R.E. Behringer, D.M. Tennant, J.E. Cunningham, M. Prentiss, K.K. Berggren, Phys. Rev. Lett. 69, 1636 (1992). doi:10.1103/PhysRevLett.69.1636

2. J.J. McClelland, R.E. Scholten, E.C. Palm, R.J. Celotta, Science 262, 877 (1993). doi:10.1126/science.262.5135.877

3. R.W. McGowan, D.M. Giltner, S.A. Lee, Opt. Lett. 20, 2535 (1995). doi:10.1364/OL.20.002535

4. R. Ohmukai, S. Urabe, M. Watanabe, Appl. Phys. B, Lasers Opt. 77, 415 (2003). doi:10.1007/s00340-003-1281-9

5. E. te Sligte, B. Smeets, K.M.R. van der Stam, R.W. Herfst, P. van der Straten, H.C.W. Beijerinck, K.A.H. van Leeuwen, Appl. Phys. Lett. 85, 4493 (2004). doi:10.1063/1.1818347

6. G. Myszkiewicz, J. Hohlfeld, A.J. Toonen, A.F.V. Etteger, O.I. Shklyarevskii, W.L. Meerts, T. Rasing, E. Jurdik, Appl. Phys. Lett. 85, 3842 (2004). doi:10.1063/1.1811804

7. R. Gupta, J.J. McClelland, Z.J. Jabbour, R.J. Celotta, Appl. Phys. Lett. 67, 1378 (1995). doi:10.1063/1.115539

8. U. Drodofsky, J. Stuhler, T. Schulze, M. Drewsen, B. Brezger, T. Pfau, J. Mlynek, Appl. Phys. B, Lasers Opt. 65, 755 (1997). doi:10.1007/s003400050342

9. E. Jurdik, G. Myszkiewicz, J. Hohlfeld, A. Tsukamoto, A.J. Toonen, A.F. van Etteger, J. Gerritsen, J. Hermsen, S. GoldbachAschemann, W.L. Meerts, H. van Kempen, T. Rasing, Phys. Rev. B 69, 201102 (2004). doi:10.1103/PhysRevB.69.201102

10. M. Mützel, S. Tandler, D. Haubrich, D. Meschede, K. Peithmann, M. Flaspöhler, K. Buse, Phys. Rev. Lett. 88, 083601 (2002). doi:10.1103/PhysRevLett.88.083601

11. D. Jürgens, A. Greiner, R. Stützle, A. Habenicht, E. te Sligte, M.K. Oberthaler, Phys. Rev. Lett. 93, 237402 (2004). doi:10.1103/PhysRevLett.93.237402

12. R. Gupta, J.J. McClelland, P. Marte, R.J. Celotta, Phys. Rev. Lett. 76, 4689 (1996). doi:10.1103/PhysRevLett.76.4689

13. D. Meschede, H. Metcalf, J. Phys. D, Appl. Phys. 36, R17 (2003). 
14. V. Balykin, P. Melentiev, Nanotechnol. Russ. 4, 425 (2009). doi:10.1134/S1995078009070040

15. M.D. Hoogerland, J.P.J. Driessen, E.J.D. Vredenbregt, H.J.L. Megens, M.P. Schuwer, H.C.W. Beijerinck, K.A.H. van Leeuwen, Appl. Phys. B, Lasers Opt. 62, 323 (1996). doi:10.1007/BF01081192

16. S. Rehse, R. McGowan, S. Lee, Appl. Phys. B, Lasers Opt. 70, 657 (2000). doi:10.1007/s003400050876

17. B. Smeets, P. van der Straten, T. Meijer, C. Fabrie, K. van Leeuwen, Appl. Phys. B, Lasers Opt. 98, 697 (2010). doi:10.1007/s00340-009-3867-3

18. J. Dalibard, C. Cohen-Tannoudji, J. Opt. Soc. Am. B 2, 1707 (1985). doi:10.1364/JOSAB.2.001707

19. R.C.M. Bosch, H.C.W. Beijerinck, P. van der Straten, K.A.H. van Leeuwen, Eur. Phys. J. Appl. Phys. 18, 221 (2002). doi:10.1051/epjap:2002042

20. B. Smeets, R. Bosch, P. van der Straten, E. te Sligte, R. Scholten, H. Beijerinck, K. van Leeuwen, Appl. Phys. B, Lasers Opt. 76, 815 (2003). doi:10.1007/s00340-003-1228-1

21. W.R. Anderson, C.C. Bradley, J.J. McClelland, R.J. Celotta, Phys. Rev. A 59, 2476 (1999). doi:10.1103/PhysRevA.59.2476
22. E. te Sligte, K.M.R. van der Stam, B. Smeets, P. van der Straten, R.E. Scholten, H.C.W. Beijerinck, K.A.H. van Leeuwen, J. Appl. Phys. 95, 1749 (2004). doi:10.1063/1.1638613

23. E. Jurdik, T. Rasing, H. van Kempen, C.C. Bradley, J.J. McClelland, Phys. Rev. B 60, 1543 (1999). doi:10.1103/ PhysRevB.60.1543

24. J. Zhong, J.C. Wells, Y. Braiman, J. Vac. Sci. Technol. B 20, 2758 (2002). doi:10.1116/1.1520558

25. T.N. Tun, M.H.T. Lwin, H.H. Kim, N. Chandrasekhar, C. Joachim, Nanotechnology 18, 335301 (2007). doi:10.1088/ 0957-4484/18/33/335301

26. R.E. Behringer, V. Natarajan, G. Timp, D.M. Tennant, J. Vac. Sci. Technol., B Microelectron. Nanometer Struct. Process. Meas. Phenom. 14, 4072 (1996). doi:10.1116/1.588647

27. A.N. Nesmeyanov, Vapor Pressure of the Chemical Elements (Elsevier, Amsterdam, 1963) 